\title{
Mixing of CNO-cycled matter in pulsationally and magnetically active massive stars
}

\author{
Norbert Przybilla ${ }^{1}$ and Maria-Fernanda Nieva ${ }^{2}$ \\ ${ }^{1}$ Dr. Remeis-Observatory \& ECAP, Sternwartstr. 7, D-96049 Bamberg, Germany \\ email: przybilla@sternwarte.uni-erlangen.de \\ ${ }^{2}$ Max-Planck-Institut für Astrophysik, Postfach 1317, D-85741 Garching, Germany \\ email: fnieva@mpa-garching.mpg.de
}

\begin{abstract}
We report on the abundances of helium, carbon, nitrogen and oxygen in a larger sample of Galactic massive stars of $\sim 7-20 M_{\odot}$ near the main sequence, composed of apparently normal objects, pulsators of $\beta$-Cephei- and SPB-type, and magnetic stars. High-quality spectra are homogeneously analysed using sophisticated non-LTE line-formation and comprehensive analysis strategies. All the stars follow a previously established tight trend in the N/C-N/O ratio and show normal helium abundances, tracing the nuclear path of the CNO-cycles quantitatively. A correlation of the strength of the mixing signature with the presence of magnetic fields is found. In conjunction with low rotation velocities this implies that magnetic breaking is highly efficient for the spin-down of some massive stars. We suggest several objects for follow-up spectropolarimetry, as the mixing signature indicates a possible magnetic nature of these stars.
\end{abstract}

Keywords. stars: abundances, stars: activity, stars: atmospheres, stars: early-type, stars: evolution, stars: magnetic fields

\section{Introduction}

Active and normal OB stars occupy the same region in the Hertzsprung-Russell diagram (HRD). While the observational characteristics of the different forms of activity are well-defined, a comprehensive understanding of the physical drivers of activity has yet to be established. Quantitative analyses of samples of active and normal stars at high precision are without doubt the starting point for progress to be made.

Two types of active OB stars are of interest in the following, pulsators of $\beta$ Cephei-type and slowly-pulsating B stars (SPBs), and magnetic stars. Their surface characteristics will be compared to those of apparently normal objects. Crucial improvements over previous work are achieved by the introduction of sophisticated modelling and a novel analysis technique, which allows in particular abundance uncertainties to be reduced to about $10-20 \%$ (1- $\sigma$ statistical), in contrast to typical values of a factor $\sim 2$ in literature.

We focus on surface abundances of $\mathrm{C}, \mathrm{N}$ and $\mathrm{O}$, which may be altered already on the main sequence due to mixing with CNO-cycled matter in the course of the evolution of rotating massive stars (e.g. Maeder \& Meynet 2000). The aim is twofold: I) to test whether mixing signatures correlate with the presence of magnetic fields (Morel et al. 2008), and II) to test whether the occurrence of pulsations is related to CNO mixing.

\section{Observations and Analysis}

Our observational sample consists of 29 bright and apparently slowly rotating early B-type stars in nearby OB associations and in the field. High-resolution $(R=40-48000)$ 


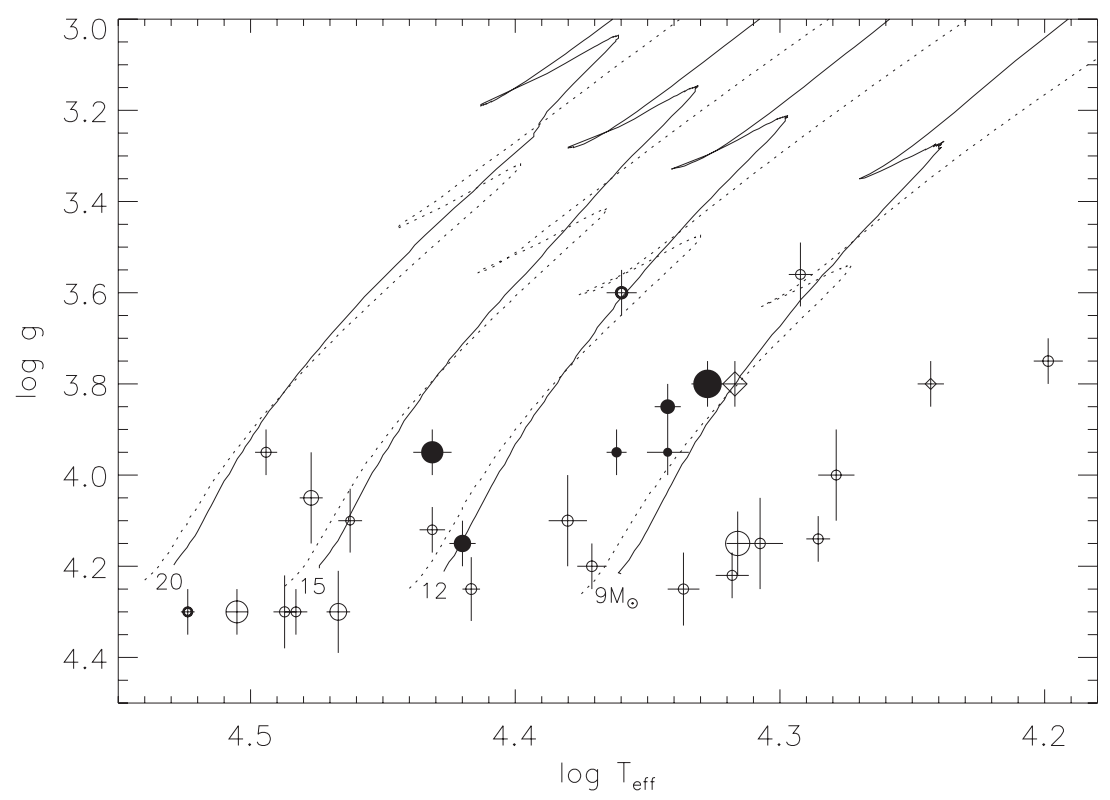

Figure 1. The sample stars in the $\log T_{\text {eff }}-\log g$-plane. Normal stars are marked by circles, $\beta$ Cep-type pulsators by dots (candidates by thick circles) and SPBs by diamonds. The symbol size encodes the $\mathrm{N} / \mathrm{C}$ mass ratio, varying between $\sim 0.3$ (cosmic value, smallest symbols) and $\sim 1.2$. Evolution tracks for rotating (full lines, $v_{\text {ini }}=300 \mathrm{~km} \mathrm{~s}^{-1}$ ) and non-rotating stars (dotted lines) are overlaid (Meynet \& Maeder 2003). The tracks were computed with $Z=0.02$, while the sample stars have $Z=0.014$, implying a downward shift of the theoretical ZAMS.

and high-S/N spectra $(S / N=250-800)$ with wide wavelength coverage were obtained with the Echelle spectrographs Foces, Feros and Fies, and from the ElOdIE archive. Details of the observations and the data reduction are discussed by Nieva \& Przybilla (in prep.) and Nieva, Simón-Díaz \& Przybilla (in prep.).

The spectra were analysed using a hybrid non-LTE approach (Nieva \& Przybilla 2007, 2008; Przybilla et al. 2008b) and a sophisticated analysis methodology. State-of-theart atomic input data were used in the modelling. In contrast to previous work, multiple hydrogen lines, the helium lines, multiple metal ionization equilibria and the stellar energy distributions were reproduced simultaneously in an iterative approach to determine the stellar atmospheric parameters. Chemical abundances for a wide range of elements were derived from analysis of practically the entire observable spectrum per element. The rewards of such a comprehensive, but time-consuming procedure were unprecedentedly small statistical error margins and largely reduced systematics (Nieva \& Przybilla 2010).

The positions of the sample stars in the $\log T_{\text {eff }}-\log g$-plane are displayed in Fig. 1. All stars are relatively unevolved objects on the main sequence in the mass range of $\sim 7-20 M_{\odot}$. While the bulk of the objects are normal stars, a small number are known pulsators, either of $\beta$ Cephei-type or SPBs. Three stars are magnetic, $\beta$ Cep (Henrichs et al. 2000), $\zeta$ Cas (Neiner et al. 2003) and $\tau$ Sco (Donati et al. 2006), one is a candidate magnetic star, $\delta$ Cet (Schnerr et al. 2008; Hubrig et al. 2009). The majority of the stars have CNO abundances compatible with cosmic values (Przybilla et al. 2008b; Nieva \& Przybilla, in prep.), about a third of the objects shows signs of mixing with CNOcycled matter. We will concentrate on this aspect and its relation to the presence of magnetic fields in the following. Fundamental parameters of the sample stars and also 


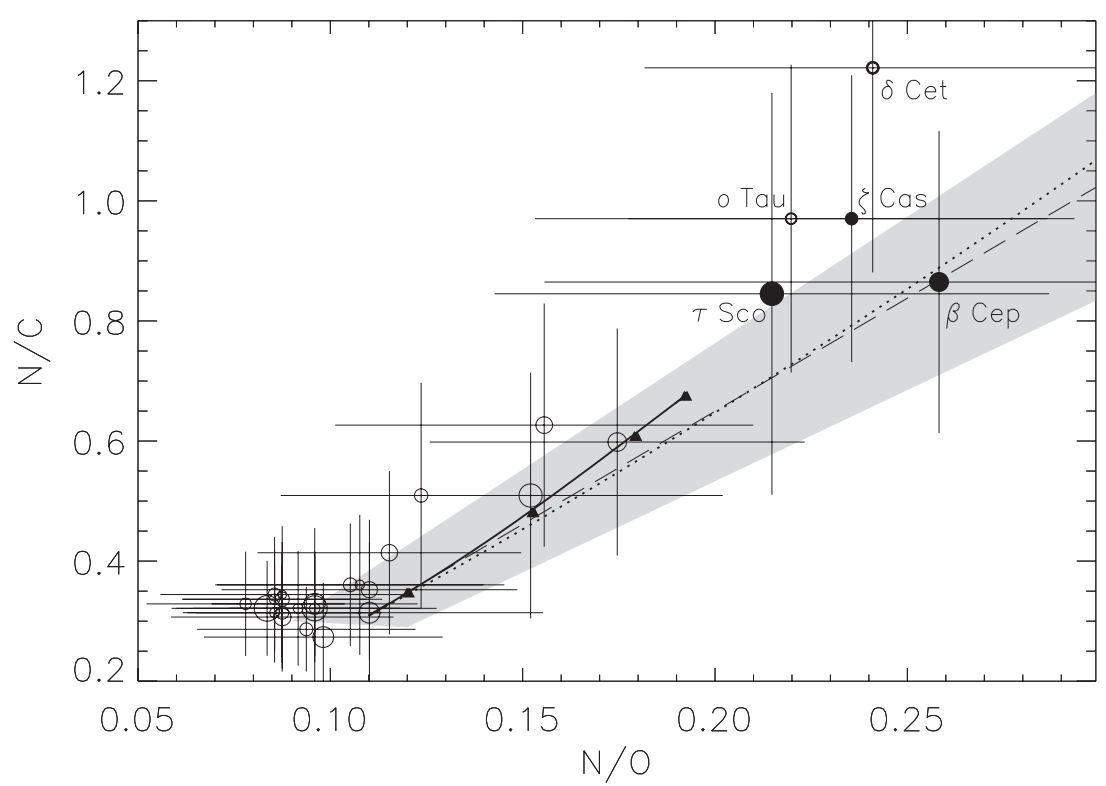

Figure 2. N/C vs. N/O abundance ratios (by mass) for our sample stars. The symbol size encodes the stellar mass, magnetic stars are marked by filled symbols (candidates by thick circles). Predictions for $9 M_{\odot}$ models are indicated by the full line, the triangles marking ratios reached at the end of core- $\mathrm{H}$ burning for $v_{\text {ini }}$ equivalent to $30,50,70$ and $90 \%$ of the star's breakup velocity (bottom to top, Ekström et al. 2008). The dotted line describes a magnetic $15 M_{\odot}$ model of Maeder \& Meynet (2005) with $v_{\text {ini }}=300 \mathrm{~km} \mathrm{~s}^{-1}$. The long-dashed line corresponds to a slope 3.77 calculated analytically for the nuclear path of the CN-cycle and initial CNO abundances as in the evolution models, while the grey area spans the full range of theoretical slopes using different references for solar abundances and the cosmic abundance standard (upper envelope).

further details on the modelling and analysis are discussed by Nieva \& Przybilla (these proceedings).

\section{Signatures of chemical mixing in early B-type stars}

Observational indications of superficial abundance anomalies for carbon, nitrogen, and oxygen (and the burning product helium) in OB-type stars on the main sequence are known for a long time. They have found a theoretical explanation by evolution models for rotating stars (e.g. Maeder \& Meynet 2000; Heger \& Langer 2000), where meridional circulation and turbulent diffusion provide the means to transport CNO-cycled matter from the stellar core to the surface. The interaction of rotation with a magnetic dynamo may increase the transport efficiency even further (Maeder \& Meynet 2005).

The changes of CNO abundances relative to each other follow a simple pattern. At the beginning of hydrogen burning, the ${ }^{14} \mathrm{~N}$ enhancement comes from the ${ }^{12} \mathrm{C}$ destruction via the $\mathrm{CN}$ cycle, and the oxygen content remains about constant. A fraction of this processed material is mixed with matter of pristine composition in the course of the subsequent evolution. A simple calculation shows that the mixing signature follows the nuclear path with slope $\mathrm{d}(\mathrm{N} / \mathrm{C}) / \mathrm{d}(\mathrm{N} / \mathrm{O}) \approx 4$ when abundances are expressed in mass fraction, see Przybilla et al. (2010) for details. Stellar evolution models follow this trend and make quantitative predictions concerning the enrichment with nuclear-processed matter. The mixing signature gets more pronounced with increasing rotational velocity and increasing mass (not shown here), and magnetic models predict the strongest mixing, see Fig. 2. The 


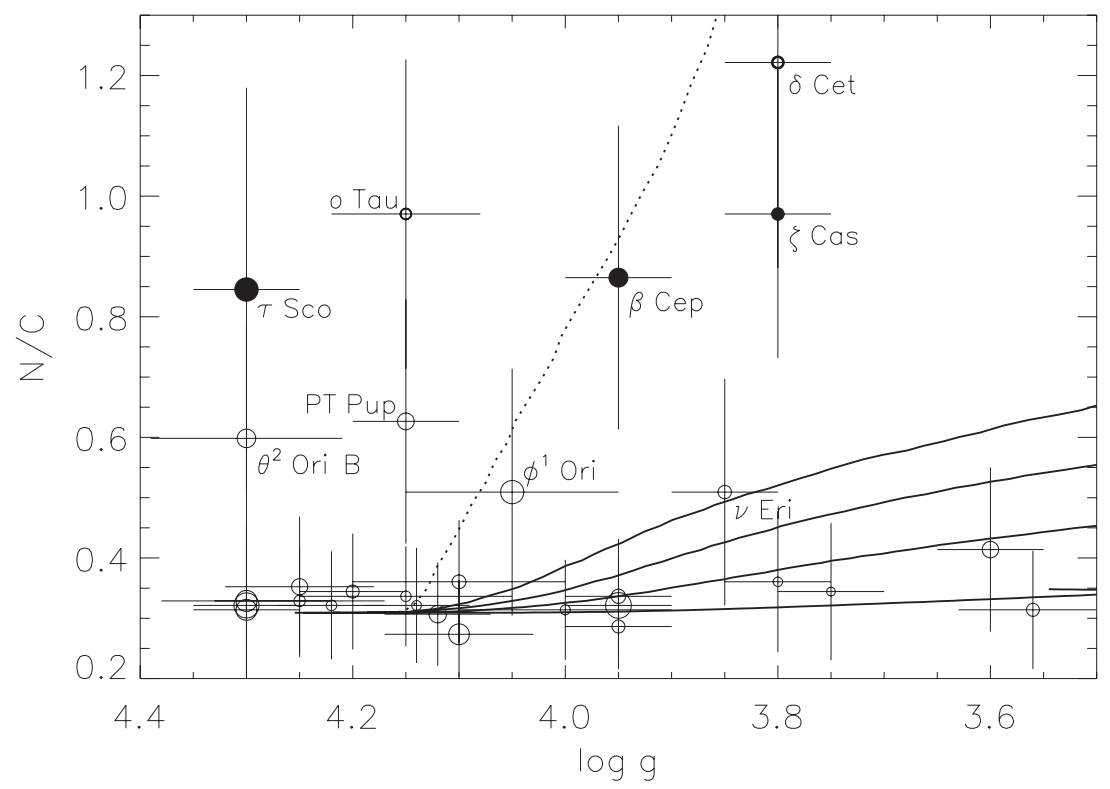

Figure 3. N/C abundance ratios on the main sequence as function of $\log g$. The same symbols as in Fig. 2 are used, the tracks for $v_{\text {ini }}$ of 30, 50, 70 and $90 \%$ breakup velocity (bottom to top) are separated in this diagram. Several additional stars with their mixing signature indicating a possible magnetic nature are also identified. The difference in metallicity between the models $(Z=0.02)$ and the sample stars $(Z=0.014)$ implies a shift of the tracks to the left in this case.

high-quality analysis of our sample stars facilitates the predicted trend to be recovered for the first time (overplotting data from previous studies would fill all areas in the N/O-N/C plane, see Fig. 1 of Przybilla et al. 2010, with the error bars exceeding the range of Fig. 2 in many cases). About half of the sample clusters around $[\mathrm{N} / \mathrm{O} \approx 0.09, \mathrm{~N} / \mathrm{C} \approx 0.32]$, which corresponds to unaltered cosmic values, several more stars show signatures apparently compatible with the rotating models, and five stars show a very high degree of mixing. Of these all but one are known (candidate) magnetic stars, suggesting a magnetic nature for the remaining one, $o$ Tau, as well. Note that the agreement between observations and theory would be even better if the models would account for cosmic $(Z=0.014$, Przybilla et al. 2008b) instead of solar abundances $(Z=0.02$, Grevesse \& Noels 1993). Helium abundances for all the sample stars are compatible with cosmic/solar values.

However, while Fig. 2 provides a crucial test for the quality of the analyses it does not facilitate to decide which models are more realistic than others, or to identify the mixing mechanism(s). Tighter constraints can be put via the $\log g$ - $\mathrm{N} / \mathrm{C}$ diagram (see Fig. 3), which relates the mixing signature to the evolutionary state of the sample stars. Indeed, the majority of objects follows the predicted trends for rotating stars, showing only mild enrichment $(\mathrm{N} / \mathrm{C} \lesssim 0.4)$ during their evolution from the zero-age main sequence (ZAMS, at the highest gravities) to the giant stage (lowest gravities). Truely fast rotators (seen pole-on) seem to be absent, which is likely a relic of one of the selection criteria for the sample assembly (stars with low $v \sin i$ ).

The magnetic stars stand out again with pronounced mixing signatures $(\mathrm{N} / \mathrm{C}>0.8)$, complemented by a group of stars at intermediate values of $\mathrm{N} / \mathrm{C} \approx 0.5-0.7$. Some of the signatures found in the more evolved magnetic stars like $\beta$ Cep and $\zeta$ Cas may be explained by evolution models accounting for a magnetic dynamo. However, these models do not predict the magnetic field to reach the stellar surface. The magnetic fields are 
probably of fossil origin instead. It is likely that the enhanced transport efficiency (when compared to models accounting for rotation only) is retained in that case, as the basic physics behind is not sensitive to the origin of the magnetic field. However, this has to be confirmed by detailed modelling.

Other objects, in particular $\tau$ Sco, challenge present stellar models seriously. The observed mixing signature of $\tau$ Sco may be explained by homogeneous evolution, but this has also to be confirmed by further computations. Homogeneous evolution would require a highly-efficient spin-down mechanism, as the star is a truely slow rotator at present (Donati et al. 2006), like several other magnetic stars with pronounced mixing signature (Morel et al. 2008). One may speculate on magnetic breaking due to angular-momentum losses by a magnetically confined line-driven stellar wind or magnetic coupling to the accretion disc during the star-forming process in the case of a fossil field. Even though the topic is not understood theoretically in a comprehensive way, spin-down times of the order of 1 Myr (Ud-Doula et al. 2009; Townsend et al. 2010) or even less (Mikulášek et al. 2008) are reported for some magnetic massive stars, possibly leading to the required slow rotation already close to the ZAMS.

After having established the connection of pronounced mixing signatures with the presence of magnetic fields, one may turn the argumentation and use the chemical fingerprint as a selection criterion for the search of magnetic fields in massive stars. Based on their mixing signatures we therefore suggest the following objects for follow-up spectropolarimetric observations: $o$ Tau as a high-potential target and $\theta^{2}$ Ori B, $\phi^{1}$ Ori, PT Pup and $\nu$ Eri (weak constraints for the latter two were presented by Hubrig et al. 2009).

Alternative explanations of the mixing signatures may be required in the case of nondetections. Case A mass-transfer in close binary systems - i.e. near the end of the core H-burning phase of the primary - is one of the most promising mechanisms. This can produce stars highly enriched in CNO-cycled products close to the ZAMS. Such stars would be fast rotators because of angular momentum transfer accompanying the mass overflow. The observational identification of such systems may be challenging, though, as the companion of the visible OB star is likely a low-mass helium star in a wide orbit, see Wellstein et al. (2001) for details.

Finally, we want to comment briefly on the question whether the occurrence of pulsations is in any way related to CNO mixing. The pulsations of $\beta$ Cep stars and SPBs are excited via the $\kappa$-mechanism because of a subsurface opacity bump due to the ionization of iron-group elements (Pamyatnykh 1999). Yet, an additional factor is required to explain the occurrence of pulsators and non-pulsators in the same regions of the HRD. Additional changes in the opacities due to modified CNO abundances as a consequence of mixing with nuclear-processed matter may be a possibility. However, our results do not support this hypothesis, pulsations occur independently of mixing signatures, see Fig. 1.

\section{Summary and Outlook}

Theory predicts surface abundances of massive stars to follow a tight relation when mixing with CNO-cycled material occurs. The nuclear path in the N/O-N/C plane is solely determined by the initial $\mathrm{CNO}$ abundances. This provides a powerful criterion for judging the quality of quantitative analyses. Few early-type massive stars can be expected to deviate from this nuclear path, either due to atomic diffusion (which is normally not operational in OB-type stars) or because of pollution with material from advanced nuclear burning phases, e.g. accreted in the course of a binary supernova (Przybilla et al. 2008a; Irrgang et al. 2010). Only observational data passing this test is well-suited for being used in the verification of different stellar evolution models. 
The high-quality analysis of our sample stars indicates that most objects can be explained well by existing models for rotating stars. It also facilitates the identification of a connection of pronounced mixing signatures with the presence of magnetic fields initially suggested by Morel et al. (2008) - at high confidence. In reverse, pronounced mixing signatures may be used to define high-potential targets for spectropolarimetric surveys that aim at the detection of magnetic stars. Several sample stars are suggested for follow-up observations.

Further studies, observational as well as theoretical, are required to develop a comprehensive understanding of chemical mixing in massive stars. High-quality analyses have to be extended to larger samples of stars, covering a wide range of rotational velocities. Further contraints to stellar evolution models can be obtained by inclusion of stars at different metallicity, like e.g. objects in the Magellanic Clouds. Once the ongoing surveys as the MiMeS project (Wade et al., these proceedings) will detect more magnetic stars it will become feasible to develop a better overview on the rôle that magnetic dynamos and/or fossil fields play in the course of the evolution of massive stars.

\section{Acknowledgements}

We would like to thank A. Maeder, G. Meynet, V. Petit, S. Simón-Díaz and G. Wade for fruitful discussion in preparation of this work. NP acknowledges travel support by the Deutsche Forschungsgemeinschaft, project number PR 685/3-1.

\section{References}

Donati, J.-F., Howarth, I. D., Jardine, M. M., Petit, P. et al. 2006, MNRAS, 370, 629

Ekström, S., Meynet, G., Maeder, A., \& Barblan, F. 2008, A\&A, 478, 467

Grevesse, N. \& Noels, A. 1993, in: N. Prantzos, E. Vangioni-Flam, \& M. Casse (eds.), Origin and Evolution of the Elements, p. 15

Heger, A. \& Langer, N. 2000, ApJ, 544, 1016

Henrichs, H. F., de Jong, J. A., Donati, J.-F., Catala, C. et al. 2000, in: M. A. Smith, H. F. Henrichs, \& J. Fabregat (eds.), IAU Colloq. 175: The Be Phenomenon in Early-Type Stars, ASP-CS 214, p. 324

Hubrig, S., Briquet, M., De Cat, P., Schöller, M. et al. 2009, AN, 330, 317

Irrgang, A., Przybilla, N., Heber, U., Nieva, M. F. et al. 2010, ApJ, 711, 138

Maeder, A. \& Meynet, G. 2000, ARAA, 38, 143

Maeder, A. \& Meynet, G. 2005, A\&A, 440, 1041

Meynet, G. \& Maeder, A. 2003, A\&A, 404, 975

Mikulášek, Z., Krtička, J., Henry, G. W., Zverko, J. et al. 2008, A\& A, 485, 585

Morel, T., Hubrig, S., \& Briquet, M. 2008, A\& A, 481, 453

Neiner, C., Geers, V. C., Henrichs, H. F., Floquet, M. et al. 2003, A\&A, 406, 1019

Nieva, M. F. \& Przybilla, N. 2007, A\& $A, 467,295$

Nieva, M. F. \& Przybilla, N. 2008, A\&A, 481, 199

Nieva, M.-F. \& Przybilla, N. 2010, in: C. Leitherer, P. Bennett, P. Morris, \& J. van Loon (eds.), Astronomical Society of the Pacific Conference Series, ASP-CS 425, p. 146

Pamyatnykh, A. A. 1999, AcA, 49, 119

Przybilla, N., Nieva, M. F., Heber, U., \& Butler, K. 2008a, ApJ (Letters), 684, L103

Przybilla, N., Nieva, M.-F., \& Butler, K. 2008b, ApJ (Letters), 688, L103

Przybilla, N., Firnstein, M., Nieva, M. F., Meynet, G. et al. 2010, A\&A, 517, A38

Schnerr, R. S., Henrichs, H. F., Neiner, C., Verdugo, E. et al. 2008, A\&GA, 483, 857

Townsend, R. H. D., Oksala, M. E., Cohen, D. H., Owocki, S. P. et al. 2010, ApJ (Letters), 714, L318

Ud-Doula, A., Owocki, S. P., \& Townsend, R. H. D. 2009, MNRAS, 392, 1022

Wellstein, S., Langer, N., \& Braun, H. 2001, A $\& A, 369,939$ 